\begin{tabular}{llll} 
Al-Rafidain Engineering & Vol.18 & No.2 & April 2010 \\
\hline
\end{tabular}

\title{
Enhancement Of Compression Zone Of Reinforced Concrete Section Due To Camber
}

\author{
Kanaan Sliwo Youkhanna Athuraia \\ College of Engineering - University of Dohuk
}

\begin{abstract}
Keywords

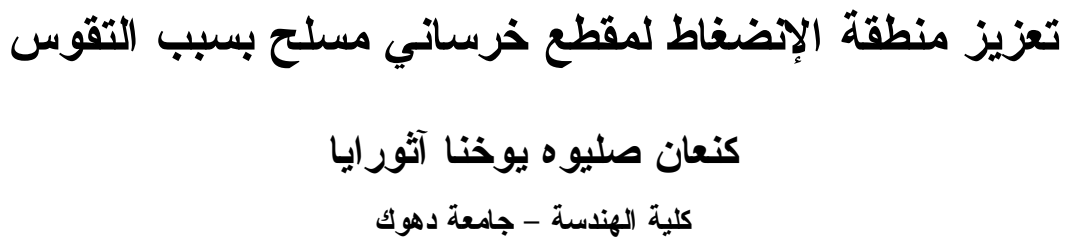

الخلاصة

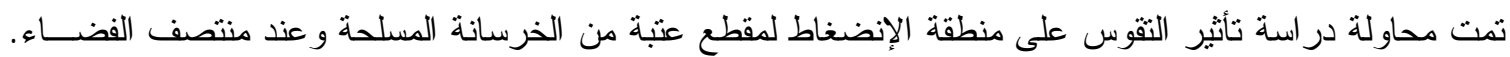

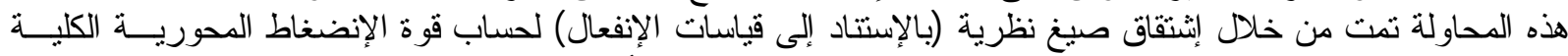
و وإجهاد الإنضغاط المحوري المؤثر على مقطع الخرسانة لهياكل خرسانية أحادية وثلاثية الفضاءاءت ذات ذات عتبـات مسـتنقيمة

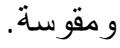


Athuraia : Enhancement Of Compression Zone Of Reinforced Concrete Section Due To ..

Introduction

[1]

[2]

3

4

Scope Of Research

Total Compressive Axial Force $\left[F_{c}\right]$

$\boldsymbol{F}_{\boldsymbol{c}}$

$$
\begin{aligned}
& \sigma_{c}=\frac{F_{c}}{A} \\
& F_{c}=\sigma_{c} \cdot A \\
& \sigma_{c}=E_{c} \cdot \varepsilon \\
& \\
& \quad F_{c}=E_{c} \cdot \varepsilon \cdot A \\
& F_{c} \\
& \sigma_{c}= \\
& A= \\
& b \\
& y
\end{aligned}
$$$$
\text { b }
$$ 


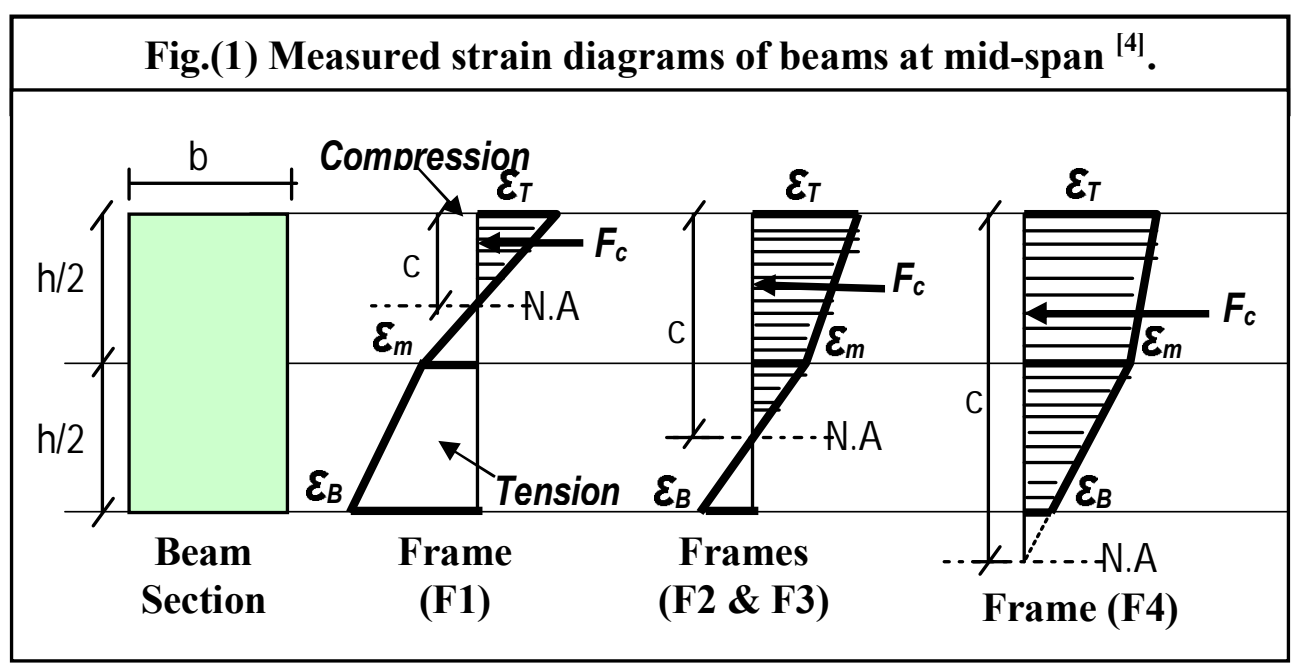

Table (1) Strain readings at mid-span ${ }^{[4]}$.

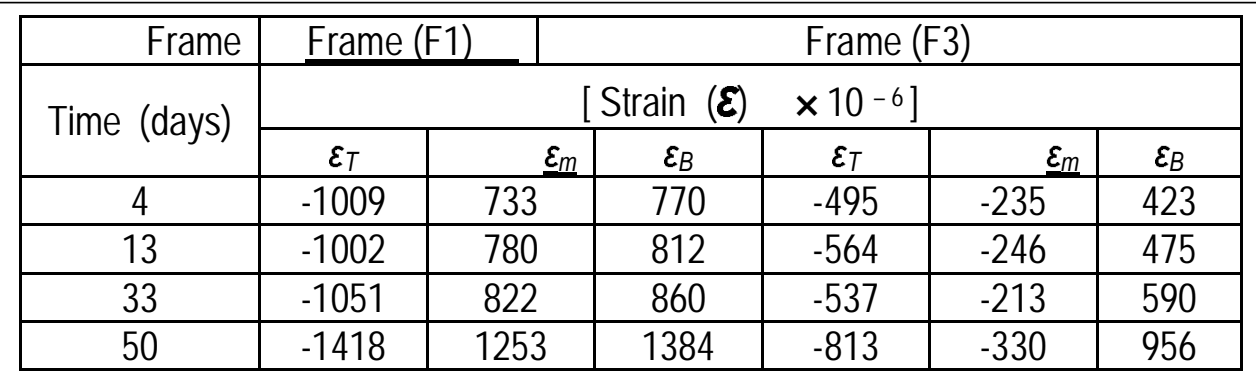

\begin{tabular}{|c|c|c|c|c|c|c|}
\hline \multirow{3}{*}{$\begin{array}{r}\text { Frame } \\
\text { Time (days) }\end{array}$} & \multicolumn{2}{|c|}{ Frame (F2) } & \multicolumn{4}{|c|}{ Frame (F4) } \\
\hline & \multicolumn{6}{|c|}{ [Strain $(\boldsymbol{\varepsilon})$} \\
\hline & $\mathcal{E}_{T}$ & $\underline{\varepsilon_{m}}$ & $\mathcal{E}_{B}$ & $\mathcal{E} T$ & $\underline{\varepsilon} m$ & $\varepsilon_{B}$ \\
\hline 4 & -1520 & -452 & 82 & -1427 & -878 & -272 \\
\hline 13 & -1610 & -469 & 92 & -1576 & -817 & -296 \\
\hline 33 & -1702 & -487 & 93 & -1662 & -980 & -316 \\
\hline 50 & -2053 & -226 & 125 & -2252 & -906 & -348 \\
\hline
\end{tabular}

$$
F_{c}
$$

$$
\begin{gathered}
F_{c}=\frac{E_{c} b \boldsymbol{h}}{4} \frac{\varepsilon_{T}{ }^{2}}{\left(\varepsilon_{T}+\varepsilon_{m}\right)} \\
F_{c}=\frac{E_{c} b h}{4}\left[\varepsilon_{T}+2 \varepsilon_{m}-\frac{\varepsilon_{B} \varepsilon_{m}}{\left(\varepsilon_{B}+\varepsilon_{m}\right)}\right]
\end{gathered}
$$


$\boldsymbol{F}_{c}=\frac{\boldsymbol{E}_{c} \boldsymbol{b h}}{4}\left(\varepsilon_{T}+2 \varepsilon_{m}+\varepsilon_{B}\right)$

Where

$$
\varepsilon_{T}, \varepsilon_{m} \quad \varepsilon_{B}
$$

$\varepsilon_{B}$

$\varepsilon_{m}$

$\varepsilon_{T}$

$\boldsymbol{h}$

b

$\boldsymbol{F}_{c}$

$E_{c}$ is the modulus of elasticity of concrete and is calculated as:

$E_{c}=4700 \sqrt{f_{c}^{\prime}}$

Table (2)

Table (1)

$\boldsymbol{F}_{\boldsymbol{c}}$

$$
\boldsymbol{F}_{\boldsymbol{c}}
$$

\begin{tabular}{|c|c|c|c|c|c|}
\hline \multicolumn{2}{|c|}{ Frame } & F1 & F2 & F3 & F4 \\
\hline \multirow{2}{*}{ Time (days) } & \multirow{2}{*}{ Load $(\mathrm{kN} / \mathrm{m})$} & \multicolumn{4}{|c|}{ Total Axial Force $\left(F_{c}\right) \quad(\mathrm{kN})$} \\
\hline & & Eq.(5) & Eq.(6) & Eq.(6) & Eq.(7) \\
\hline 4 & \multirow{3}{*}{2.533} & 10.69 & 43.07 & 14.36 & 63.20 \\
\hline 13 & & 11.80 & 45.20 & 15.86 & 64.13 \\
\hline 33 & & 10.79 & 47.52 & 14.49 & 72.04 \\
\hline 50 & 4.576 & 13.77 & 44.35 & 22.07 & 80.71 \\
\hline
\end{tabular}

Table (2) Total compressive axial force $\left(F_{c}\right)$.

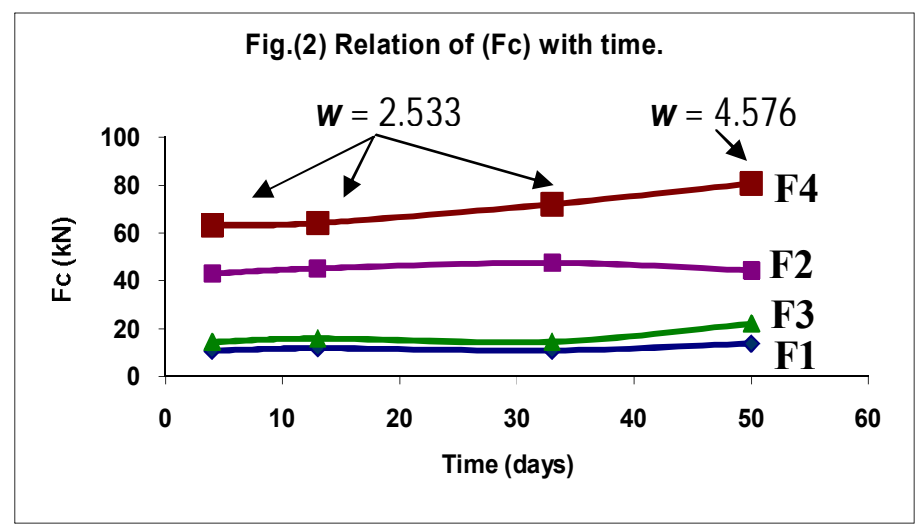


Al-Rafidain Engineering

Vol.18

No.2

April 2010

5

4

Axial Compressive Stress $\left(\Sigma_{c}\right)$

$\sigma_{c}$

$\sigma_{c}=\boldsymbol{E}_{c} \cdot \varepsilon$

$\varepsilon$
(8)

and $\quad \varepsilon_{2} \frac{\varepsilon_{m} \varepsilon_{B}}{2}$ 
Hence $\quad \sigma_{c}=E_{c}\left(\frac{\varepsilon_{T}+2 \varepsilon_{m}+\varepsilon_{B}}{4}\right)$

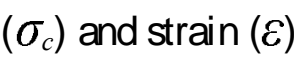

$\sigma_{c}$

$$
\begin{array}{r}
\sigma_{c} \\
\sigma_{c}=\frac{\boldsymbol{F}_{c}}{\boldsymbol{b h}}
\end{array}
$$

$F_{c} \sigma_{c}$

$b h$ $F_{c}$

Table (3) Axial compressive strain and stress.

\begin{tabular}{|c|c|c|c|c|c|}
\hline \multicolumn{2}{|c|}{ Frame } & \multicolumn{2}{c|}{ F3 } & \multicolumn{2}{c|}{ F4 } \\
[Triple Straight] & \multicolumn{2}{c|}{ [Triple Camber] } \\
\hline $\begin{array}{c}\text { Time } \\
\text { (days) }\end{array}$ & $\begin{array}{c}\text { Load } \\
(\mathrm{kN} / \mathrm{m})\end{array}$ & $\begin{array}{c}\varepsilon \\
\times 10^{-6}\end{array}$ & $\begin{array}{c}\sigma_{c} \\
\mathrm{~N} / \mathrm{mm}^{2}\end{array}$ & $\begin{array}{c}\varepsilon \\
\times 10^{-6}\end{array}$ & $\begin{array}{c}\sigma_{c} \\
\mathrm{~N} / \mathrm{mm}^{2}\end{array}$ \\
\hline 4 & & 585.50 & 14.28 & 863.75 & 21.07 \\
\cline { 4 - 7 } & \multirow{2}{*}{2.533} & 614.00 & 14.98 & 876.50 & 21.38 \\
\cline { 3 - 6 } & 645.75 & 15.75 & 984.50 & 24.01 \\
\hline 33 & 4.576 & 595.00 & 14.51 & 1103.00 & 26.90 \\
\hline 50 & 2.576 & & & & \\
\hline
\end{tabular}

Fig.(3) Relationship between and time.

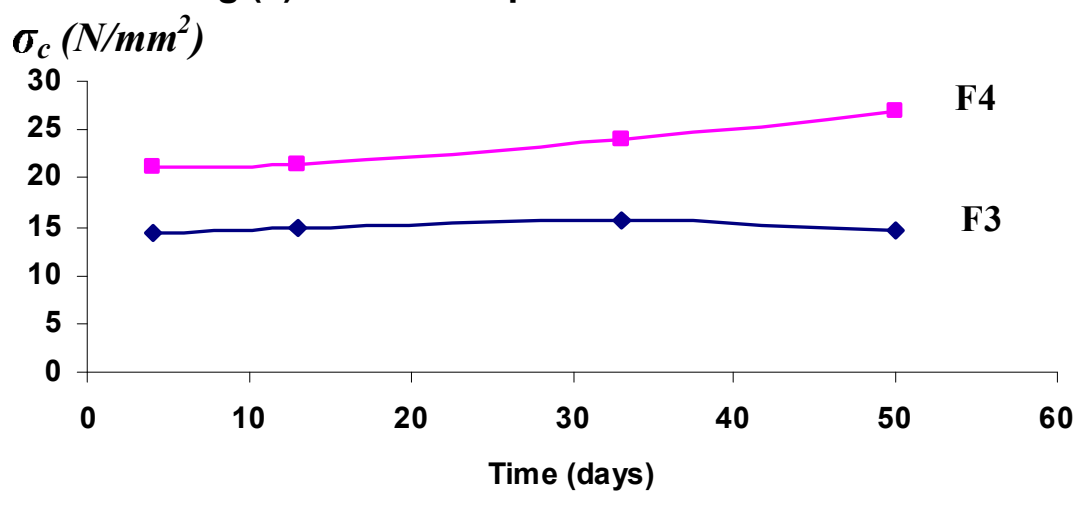




\begin{tabular}{llll} 
Al-Rafidain Engineering & Vol.18 & No.2 & April 2010 \\
\hline
\end{tabular}

\section{Conclusions}

\section{References}

1.

2.

3.

4.

5.

6 ندوة تحقيق شروط الراحة والأمان في الأبنية - كلية الهندسة الميكانيكية والكهربائية - جامعة البعث - حمص سوريا، بالتعاون مع إتحاد مجالس البحث العلمي العربية ، 2002/10/28-26.

Appendix (A)
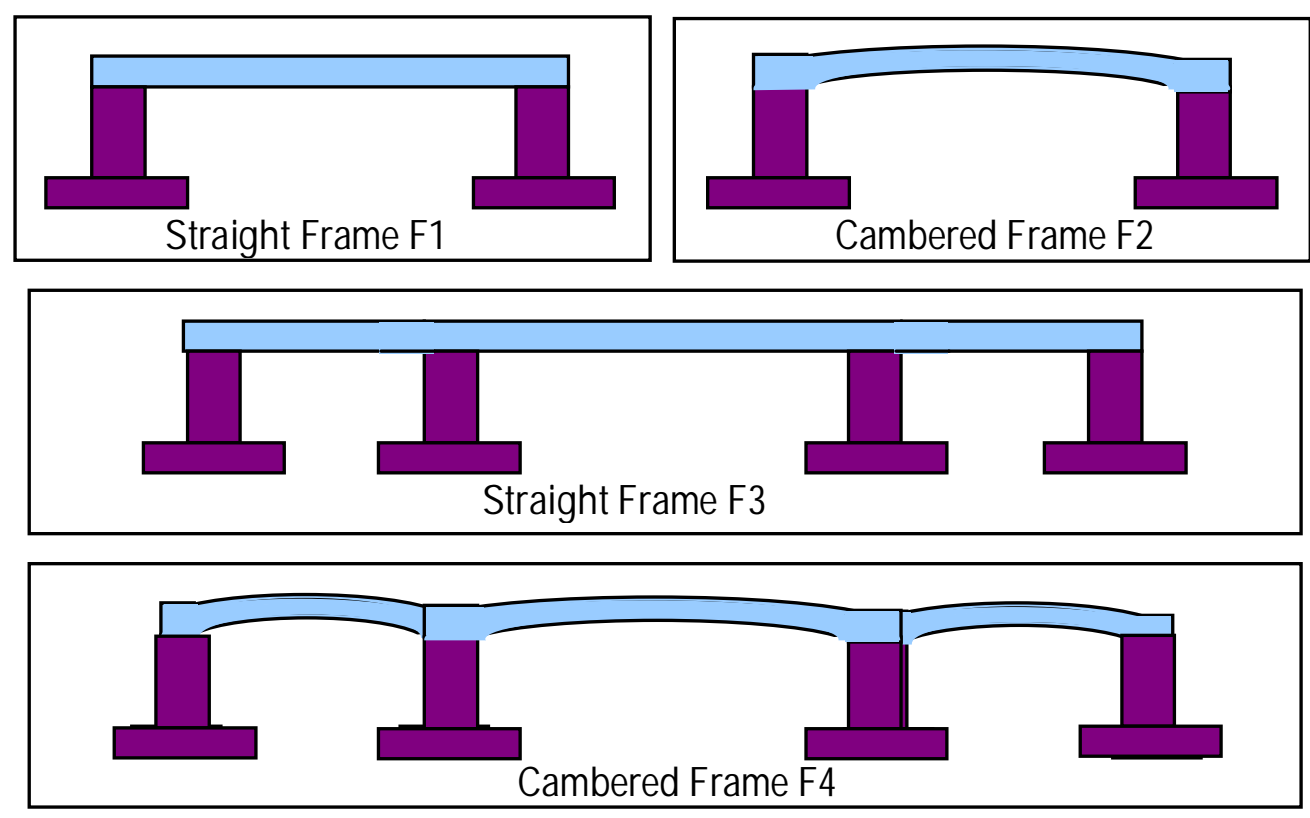